\title{
Evaluation of medical and psychological parameters of quality of life in supraventricular tachyarrhythmia children. A comparison with healthy children
}

Emilia Szafran¹, Artur Baszko ${ }^{1}$ Anna Bukowska-Posadzy², Tomasz Moszura ${ }^{1,3}$, Bożena Werner ${ }^{4}$, Aldona Siwińskaํㅡ. Maciej Banach ${ }^{5}$, Jarosław Walkowiak², Waldemar Bobkowski ${ }^{1}$

\author{
${ }^{1}$ Department of Pediatric Cardiology, Poznan University of Medical Sciences, Poznan, \\ Poland \\ ${ }^{2}$ Department of Pediatric Gastroenterology and Metabolic Diseases, Poznan University \\ of Medical Sciences, Poznan, Poland \\ ${ }^{3}$ Department of Cardiology, Polish Mother's Memorial Hospital - Research Institute, \\ Lodz, Poland \\ ${ }^{4}$ Department of Pediatric Cardiology and General Pediatrics, Warsaw University \\ of Medicine, Warsaw, Poland \\ ${ }^{5}$ Department of Hypertension, WAM University Hospital, Medical University of Lodz, \\ Lodz, Poland
}

Submitted: 29 January 2016

Accepted: 26 June 2016

Arch Med Sci 2016; 12, 5: 1052-1063

DOI: 10.5114/aoms.2016.61912

Copyright (c) 2016 Termedia \& Banach

\section{Abstract}

Introduction: There are only a few available studies evaluating quality of life $(\mathrm{QoL})$ in pediatric patients with cardiac arrhythmia. The aim of the study was to evaluate medical and psychological parameters of the QoL in children with a diagnosed supraventricular tachyarrhythmia (SVT) and to compare the obtained data with a group of healthy children (HC).

Material and methods: Inclusion criteria: children aged 7-18 with SVT, treated at Poznan University of Medical Sciences, Department of Pediatric Cardiology. The evaluation tools were the WHOQOL-BREF instrument and a questionnaire related to the patient's feelings and observations concerning arrhythmia (Pediatric Arrhythmia Related Score - PARS), developed by the authors and adjusted to the group of arrhythmia patients.

Results: The study included 180 SVT children and $83 \mathrm{HC}$. On the basis of WHOQOL-BREF the SVT group was found to have lower assessment values of QoL within the physical domain (Phd) (mean \pm SD: $65.7 \pm 15.8$ vs. $81.6 \pm 12.8$; $p<0.0001)$ and psychological domain (Psd) (mean \pm SD: $75.8 \pm 15.2$ vs. 81.3 $\pm 14.1 ; p<0.005)$. No significant differences were found within the social relationships domain or the environment domain. On the basis of PARS in the SVT group the patients reported significantly increased symptoms within Phd (mean \pm SD: $2.3 \pm 0.7$ vs. $1.6 \pm 0.3 ; p<0.0001$ ) as well as increased negative feelings within Psd (mean \pm SD: $2.3 \pm 0.7$ vs. $2.1 \pm 0.6 ; p<0.005$ ). Conclusions: Medical and psychological parameters of the QoL in SVT children are significantly lower in comparison with HC. A diagnosis of SVT has no influence on the social and environmental areas of QoL. The PARS appears to be a useful tool to supplement the generic questionnaire for QoL evaluation in SVT children.

Key words: quality of life, arrhythmia, questionnaires, pediatric.

\author{
Corresponding author: \\ Emilia Szafran MD \\ Department \\ of Pediatric Cardiology \\ Poznan University \\ of Medical Sciences \\ 27/33 Szpitalna St \\ 60-149 Poznan, Poland \\ Phone: +48503474153 \\ E-mail: emi101@wp.pl
}




\section{Introduction}

The most common type of symptomatic tachyarrhythmia in children is supraventricular tachycardia (SVT) [1, 2]. Supraventricular tachycardia in children is rarely a life-threatening condition; however, it may produce many symptoms, such as palpitations, dizziness, syncope, chest pain, dyspnea, and lower exercise tolerance, and consequently impede the comfort of life [1, 3]. The symptoms and consequences of arrhythmia depend, among other things, on its type, frequency of cardiac rhythm or its duration.

In the pediatric group the accessory atrioventricular pathway, due to Wolff-Parkinson-White syndrome, is the most frequent cause of SVT. A less common type of SVT is atrioventricular nodal reentrant tachycardia (AVNRT) or atrial ectopic tachycardia (AET) [1, 2]. The present studies carried out among adult patients prove that cardiac arrhythmias may significantly affect the quality of life (QoL) $[4,5]$. The results of studies performed in pediatric patients also indicate that arrhythmia may significantly impact the QoL $[3,6,7]$. There are only a few available studies evaluating the quality of life in pediatric patients with SVT. Quality of life may be difficult to evaluate among pediatric patients as it must be multidimensional and involve physical, mental, and social health domains. In accordance with the current recommendations QoL evaluation must be performed on the basis of a generic questionnaire supplemented by a specific questionnaire for the studied group. Until recently, researchers investigating the QoL in children with cardiac conditions based their studies only on generic questionnaires, which may be useful but not thoroughly effective to evaluate the QoL of children with arrhythmia or heart disease [8]. Therefore, in recent years several pediatric questionnaires have been developed, designed for cardiac patients, such as the PedsQL Cardiac Module $[9,10]$ or the Pediatric Cardiac Quality of Life Inventory [11-13]. Unfortunately, neither of these is available in a Polish language version.

The aim of this study was to evaluate the medical and psychological parameters of the QoL of the children with a confirmed diagnosis of SVT and to compare the obtained results with a group of healthy children. The evaluation was done on the basis of the generic questionnaire supplemented by the specific questionnaire developed by the authors for the needs of the studied group.

\section{Material and methods}

\section{Patients}

The study group included SVT children aged 7-18, inhabitants of Poland, with no organic heart disease or other chronic conditions that may dis- turb the QoL, treated in our institution during 20102014. The control group was composed of healthy children, aged 7-18, inhabitants of Poland, with no medical conditions that could impede their QoL and who took no medicines. This group was selected by pediatricians, who were acquainted with the authors, during routine check-ups in 2014-2015. The minimal inclusion criterion was the age of 7 years, which was perceived as the age of school maturity. It was assumed that children below 7 years of age might find it difficult to provide autonomous, or with a little help from adults, answers to all the questions in the questionnaire and, what is more, before 6 years of age emotional lability, treated as a developmental norm, could negatively affect the reliability of the given answers.

\section{WHOQOL-BREF (Appendix 1)}

The WHOQOL-BREF instrument comprises 26 items, which measure the following broad domains: physical health, psychological health, social relationships, and environment. The WHOQOLBREF is a shorter version of the original instrument that may be more convenient for use in large research studies or clinical trials [14]. The questions included in the questionnaire are rated on a five-point Likert scale; the points are calculated in accordance with the established code and transformed to a 4-20 scale and subsequently to a 0-100 scale. The higher the score obtained in one domain, the higher is the QoL. WHOQOL-BREF also includes questions analyzed individually: question 1 , concerning individual general perception of QoL, and question 2, concerning individual general perception of one's health. Considering the patients' age, the question regarding sexual activity, part of the social domain, was removed from the questionnaire.

\section{Pediatric Arrhythmia Related Score (Appendix 2)}

The second instrument used in this study was the questionnaire regarding patients' feelings and observations connected with arrhythmia - the Pediatric Arrhythmia Related Score (PARS), which was developed for the purpose of the scientific project by pediatric cardiologists in collaboration with a clinical psychologist and adjusted to the group of arrhythmia children. The questionnaire contains 32 questions, which are grouped into 3 domains: physical, regarding the symptoms perceived as specific or likely to accompany SVT; medical satisfaction, concerning cooperation with medical care professionals; and the psychological domain, referring to the emotional condition of the studied individuals. The answers are provided using a 1-5 point scale where ' 1 ' means 'absolutely 
not' and ' 5 ' means 'absolutely yes'. Each domain is assessed on a 1-5 point scale and numeric results of individual areas are 'negatively directed', i.e. the lower the numeric value, the higher the QoL. For the purpose of this research, only the questions regarding physical and psychological aspects were used, assuming that the questions referring to medical satisfaction are inappropriate to be analyzed in a group of healthy children, who do not or hardly have any contacts with medical care professionals. However, these questions were used for further analyses of the group of SVT children.

\section{Data collection}

The WHOQOL-BREF questionnaire and the unabbreviated PARS questionnaire were completed by both groups of patients.

The inclusion criteria for a patient were to provide written consent from their parents and the patient's approval after they had become familiar with all the data regarding the scientific study. On entering the study each patient underwent a detailed interview and physical examination. The effects of cardiac arrhythmias on medical and psychological parameters of the QoL were assessed in patients with SVT included in the study, and the collected results were compared with the group of healthy children. The study protocol was approved by the Bioethics Committee.

\section{Statistical analysis}

Statistica 10 (a data analysis software system) was used to perform the statistical analysis. A probability level of $p<0.05$ was regarded as significant. The two groups were statistically compared with regard to age, gender, education and place of living (village/town). Due to the lack of a normal distribution, the comparison of patients' age was performed using the nonparametric Mann-Whitney $U$ test. The age was determined by a mean value, standard deviation and a median. Gender and place of living were compared using the $\chi^{2}$ with Yates correction test. The above results were presented as numbers in categories and percentage values. Education was compared using a nonparametric Mann-Whitney $U$ test, and the results were also presented as numbers in categories and percentage values. The study compared the scores obtained in 4 domains of the WHOQOL-BREF questionnaire and 2 domains of the PARS questionnaire of SVT children and healthy children. Moreover, two questions of WHOQOL-BREF were analyzed individually: question 1, concerning individual general perception of the QoL, and question 2, concerning individual general perception of one's health. The above values were represented using the mean and stan- dard deviation, median and range. The nonparametric Mann-Whitney $U$ test was used to perform comparisons of the above quality of life parameters between the two groups of patients. Comparisons in the SVT group were also performed. The patients who take medicines and the patients who do not take them were compared using the nonparametric Mann-Whitney $U$ test. The analysis was also performed to evaluate how the QoL of SVT children differs depending on arrhythmia duration (from diagnosis until the time of questionnaire completion), SVT type (atrioventricular reentrant tachycardia - AVRT, AVNRT, AET) and frequency of SVT episodes (at least once a month, less frequently than once a month but at least once every 3 months, and patients with SVT episodes less frequently than once every 3 months). For these analyses the nonparametric KruskalWallis test with the Dunn-Bonferroni multiple comparison test were used. To assess correlations between scores in PARS and WHOQOL-BREF, Spearman's rank correlation coefficient was used.

\section{Results}

\section{Patient demographics}

All children with cardiac disorders included in the study underwent electrophysiological study (EPS), on the basis of which the following forms of SVT were diagnosed: AVRT (61.1\%), AVNRT (33.3\%), AET (3.9\%). One child was diagnosed with persistent junctional reciprocating tachycardia (PJRT). In 2 patients with SVT, previously confirmed with ECG, arrhythmias were not induced during the EPS study.

The mean \pm SD age of the first SVT episode was $10 \pm 4.6$ years. In the SVT group clinical symptoms were reported by $89.4 \%$ of children, while the remaining patients were asymptomatic. In the SVT group antiarrhythmic drugs were taken by $64.4 \%$ of the patients, while the remaining children were not treated pharmacologically (Table I).

\section{WHOQOL-BREF}

The analysis of the provided responses on a five-point Likert scale showed differences between the group of healthy children and SVT patients. Significant differences were found regarding general satisfaction with the quality of life $(p<0.0001)$ and general satisfaction with the health condition ( $p<0.0001)$ : only 1 child $(1.2 \%)$ of the healthy group was dissatisfied with the quality of life (mean: 4.3; median: 4.0), whereas dissatisfaction was expressed by $12.7 \%$ of the children in the SVT group (mean: 3.8 ; median: 4.0); similarly, $24.6 \%$ of arrhythmia children were dissatisfied with their general health condition (mean: 3.1; median: 3.0), while only one patient in 
Table I. Patient demographics

\begin{tabular}{|lccc|}
\hline Parameter & SVT children group & Healthy children group & $P$-value \\
\hline Patient, $n$ & 180 & 83 & \\
\hline Age, mean \pm SD (median) [years] & $13.7 \pm 3.0(14)$ & $38(45.8)$ & 0.1496 \\
\hline Gender, $n$ (\%): & $91(50.6)$ & $45(54.2)$ & 0.5573 \\
\hline Boys & $89(49.4)$ & & 0.4419 \\
\hline Girls & & $23(27.7)$ & \\
\hline Place of living, $n$ (\%): & $60(33.3)$ & $60(72.3)$ & \\
\hline Village & $120(66.7)$ & & 0.3460 \\
\hline Town & & $35(42.2)$ & \\
\hline Education, $n$ (\%): & $56(31.1)$ & $22(26.5)$ & $1(1.2)$ \\
\hline Primary school & $67(37.2)$ & $6(7.2)$ & \\
\hline Secondary school & $3(1.7)$ & $19(22.9)$ & \\
\hline Basic vocational school & $16(8.9)$ & $38(21.1)$ & \\
\hline Technical college & & & \\
\hline Higher education & & & \\
\hline
\end{tabular}

the group of healthy children expressed dissatisfaction (mean: 4.2; median: 4.0) (Figure 1).

Following the analysis of the WHOQOL-BREF questionnaire it was observed that the SVT group demonstrated significant differences in comparison with healthy children with regard to physical and psychological domains. The lowest scores were found on the following sub-scales regarding physical domain (Phd): pain and discomfort, dependency on drugs and treatment, energy and fatigue, mobility, everyday activities, work capacity (Table II). The number of points on a 0-100 scale obtained in Phd in the SVT group was mean \pm SD: $65.7 \pm 15.8$, whereas in the healthy children it was $81.6 \pm 12.8$ (Figure 2). Significant differences were also found regarding the following sub-scales that refer to the psychological domain (Psd): self-esteem, negative feelings, memory and concentration (Table II). The average number of points in Psd was mean \pm SD: $75.8 \pm 15.2$ in the SVT group and $81.3 \pm 14.1$ in the healthy children (Figure 2 ).

No significant differences were found with regard to the average number of points in the following domains: social relationships (SRD) and environment (EnD). The average number of points in SRD was mean \pm SD: $79.2 \pm 16.8$ in the SVT group and $80.6 \pm 16.5$ in the healthy children; the average number of points in EnD was mean \pm SD: $77.4 \pm 13.3$ in the SVT group and $79.3 \pm 15.5$ in the healthy children (Figure 2, Table II).
A

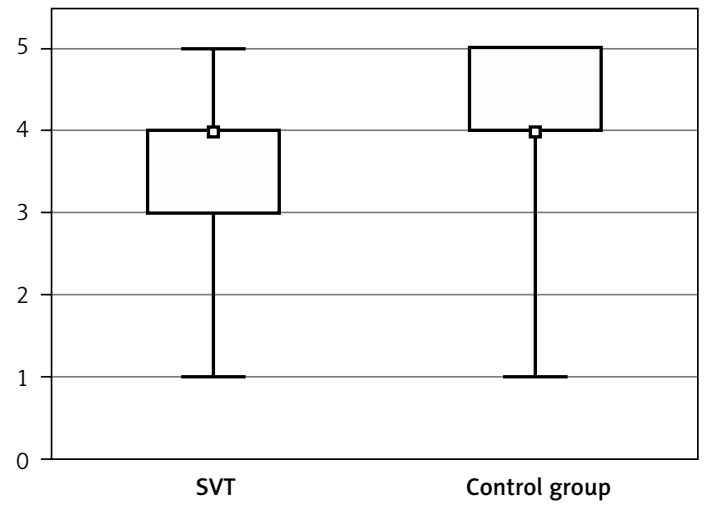

B

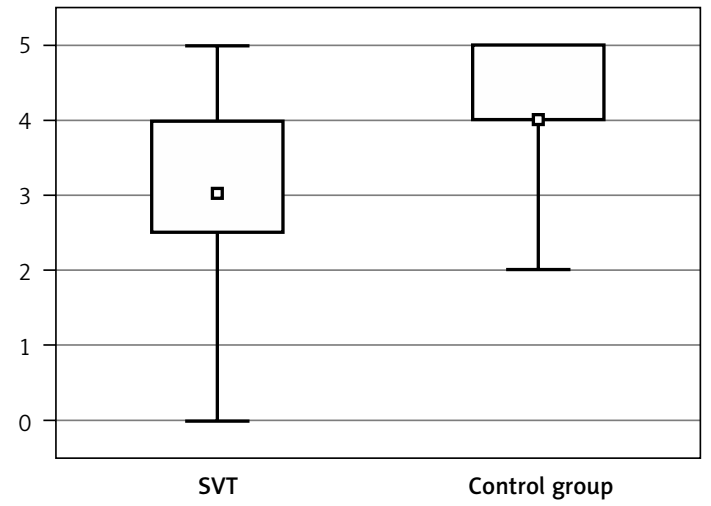

a Median $\square 25-75 \% \quad$ IMin.-max.

Figure 1. General satisfaction with the quality of life (A) and with the health condition (B). Comparison of SVT children and healthy children 
E. Szafran, A. Baszko, A. Bukowska-Posadzy, T. Moszura, B. Werner, A. Siwińska, M. Banach, J. Walkowiak, W. Bobkowski

Table II. Sub-scales of WHOQOL-BREF assessment. Comparison of SVT children and healthy children

\begin{tabular}{|c|c|c|c|c|c|}
\hline \multirow[t]{2}{*}{ Sub-scales } & \multicolumn{2}{|c|}{ SVT children group } & \multicolumn{2}{|c|}{ Healthy children group } & \multirow[t]{2}{*}{$P$-value } \\
\hline & Mean & Median & Mean & Median & \\
\hline \multicolumn{6}{|l|}{ Physical domain: } \\
\hline Pain and discomfort & 3.6 & 4.0 & 4.2 & 5.0 & $<0.0001$ \\
\hline Dependency on drugs and treatment & 3.3 & 3.0 & 4.8 & 5.0 & $<0.0001$ \\
\hline Mobility & 3.5 & 4.0 & 4.2 & 4.0 & $<0.0001$ \\
\hline Work capacity & 3.7 & 4.0 & 4.1 & 4.0 & 0.0001 \\
\hline Energy and fatigue & 3.9 & 4.0 & 4.3 & 4.0 & $<0.0001$ \\
\hline Activities of daily living & 3.6 & 4.0 & 4.2 & 4.0 & $<0.0001$ \\
\hline Sleep and rest & 3.9 & 4.0 & 4.0 & 4.0 & 0.2739 \\
\hline \multicolumn{6}{|l|}{ Psychological domain: } \\
\hline Self-esteem & 4.1 & 4.0 & 4.3 & 4.0 & 0.0058 \\
\hline Negative feelings & 3.8 & 4.0 & 4.1 & 4.0 & 0.0167 \\
\hline Thinking, learning, memory and concentration & 3.9 & 4.0 & 4.1 & 4.0 & 0.0377 \\
\hline Positive feelings & 4.0 & 4.0 & 4.2 & 4.0 & 0.0679 \\
\hline Bodily image and appearance & 4.0 & 4.0 & 4.3 & 5.0 & 0.0680 \\
\hline Meaning of life & 4.3 & 5.0 & 4.4 & 5.0 & 0.2170 \\
\hline \multicolumn{6}{|l|}{ Social relationships domain: } \\
\hline Personal relationships & 4.1 & 4.0 & 4.2 & 4.0 & 0.2269 \\
\hline Social support & 4.2 & 4.0 & 4.2 & 4.0 & 0.9475 \\
\hline \multicolumn{6}{|l|}{ Environment domain: } \\
\hline Financial resources & 3.9 & 4.0 & 4.0 & 4.0 & 0.2315 \\
\hline Freedom, physical safety and security & 4.3 & 4.0 & 4.4 & 5.0 & 0.1222 \\
\hline Health and social care & 3.6 & 4.0 & 3.4 & 3.0 & 0.0518 \\
\hline Home environment & 4.5 & 5.0 & 4.4 & 5.0 & 0.7106 \\
\hline Opportunities for acquiring new information and skills & 4.4 & 5.0 & 4.5 & 5.0 & 0.1865 \\
\hline Opportunities for recreation/leisure activities & 3.9 & 4.0 & 4.3 & 5.0 & 0.0019 \\
\hline Physical environment (pollution/noise/traffic/climate) & 4.0 & 4.0 & 4.1 & 4.0 & 0.4051 \\
\hline Transport & 3.7 & 4.0 & 3.7 & 4.0 & 0.8840 \\
\hline
\end{tabular}

\section{PARS (authors' own questionnaire)}

The analysis of the PARS questionnaire showed significant differences in both compared domains (Figure 3). The mean \pm SD value on a $1-5$ scale in Phd was $2.3 \pm 0.7$ in the SVT group and $1.6 \pm 0.3$ in the control group. The mean \pm SD value in Psd was $2.3 \pm 0.7$ in the SVT group and $2.1 \pm 0.6$ in the healthy children.

The analysis of Phd showed significant differences in 11 out of 13 questions related to the discussed domain, indicating significantly increased symptoms in the SVT group (Table III); Psd showed differences in 3 out of 7 related questions. The SVT patients were more likely to cry and feel sad and nervous when compared with the healthy children (Table III).

\section{SVT group analysis}

In the SVT group the patients who take medicines and those who do not were compared. The children who take medicines were less satisfied with their general health condition (mean/median: 3.0/3.0 vs. 3.4/4.0; $p=0.0017$ ) and ranked lower only in Phd of the WHOQOL-BREF question- 
A

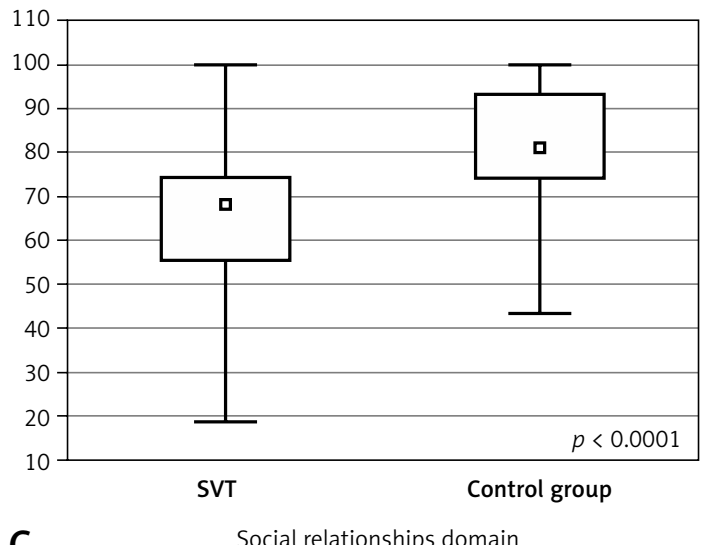

C

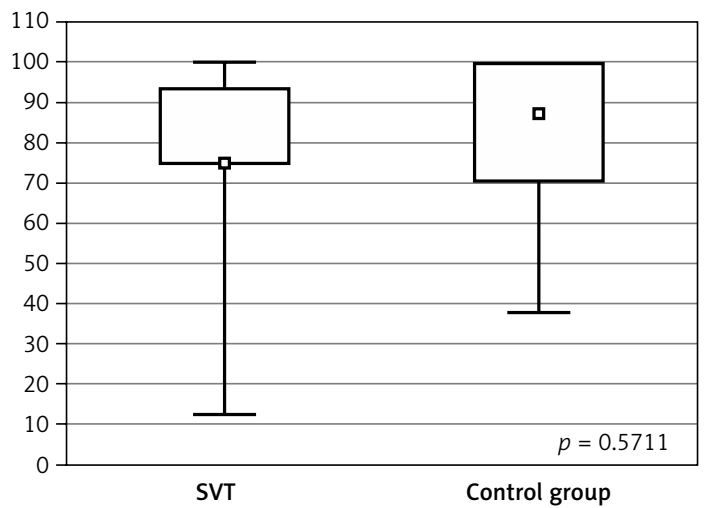

B

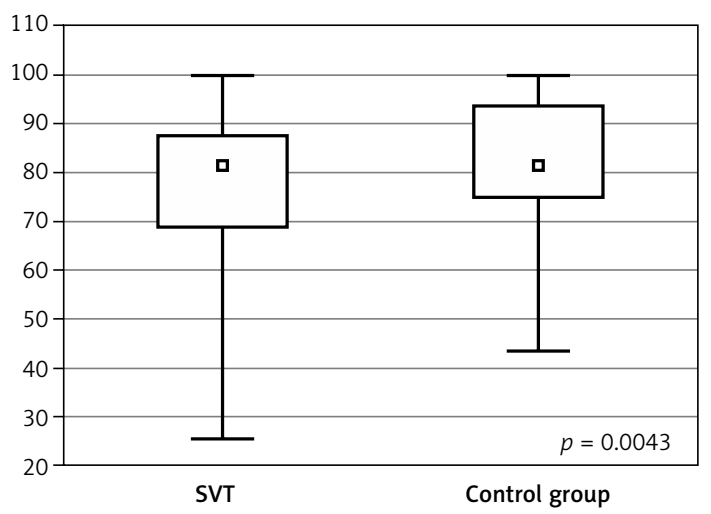

D

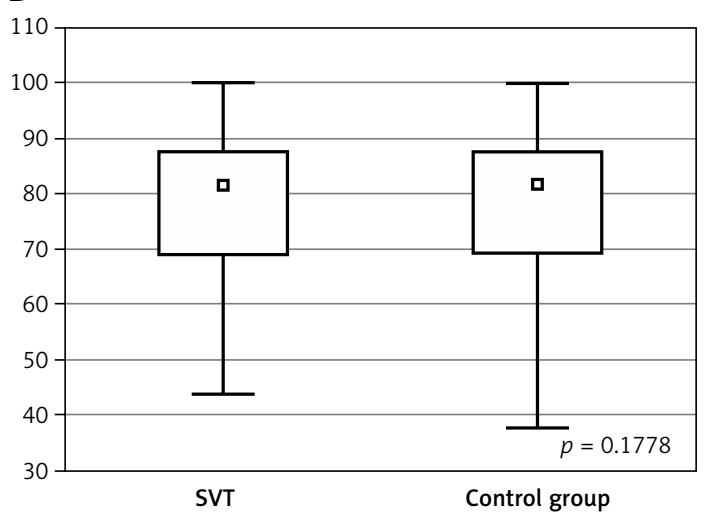

- Median

$25-75 \%$

I Min.-max.

Figure 2. WHOQOL-BREF scores. Comparison of SVT children and healthy children

A

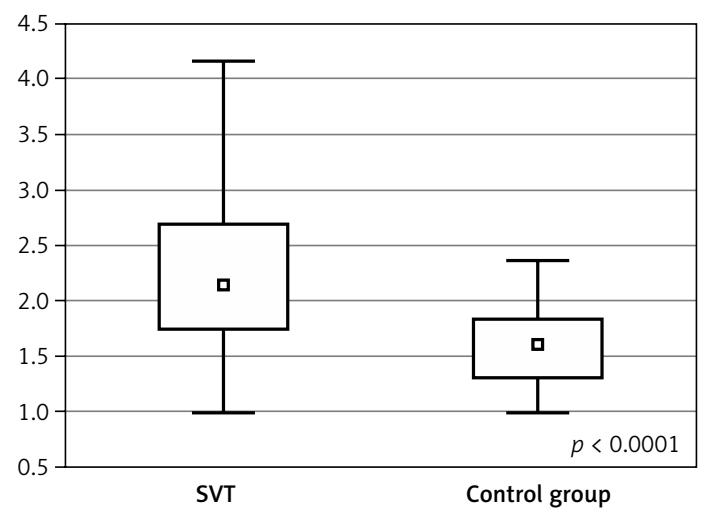

B



a Median $\square 25-75 \% \quad$ IMin.-max.

Figure 3. PARS scores. Comparison of SVT children and healthy children

naire (mean \pm SD/median: $63.4 \pm 15.8 / 68.8$ vs. $69.8 \pm 15.1 / 68.8 ; p=0.0288)$.

Frequency of SVT episodes had a significant impact on the QoL. The differences were observed among children who had SVT episodes at least once a month and the patients with SVT episodes less frequently than once every 3 months: the frequency of SVT episodes affected the general satisfaction with the QoL (mean/ median: 3.6/4.0 vs. 4.0/4.0; $p=0.0143$ ) and general satisfaction with the health condition (mean/median: $2.8 / 3.0$ vs. $3.5 / 3.0 ; p=0.0016$ ). Significant differences were also observed in Phd of the PARS questionnaire (mean $\pm \mathrm{SD}$ /median: $2.4 \pm 0.7 / 2.3$ vs. $2.1 \pm 0.7 / 2.0 ; p=0.0159)$ and $\mathrm{Phd}$ of the WHOQOL-BREF questionnaire (mean \pm SD/ median: $63.2 \pm 15.7 / 62.5$ vs. $70.1 \pm 16.0 / 68.8$; $p=0.0181)$. 
Table III. PARS questions and scores. Comparison of SVT children and healthy children

\begin{tabular}{|c|c|c|c|c|c|}
\hline \multirow[t]{2}{*}{ Questions } & \multicolumn{2}{|c|}{ SVT children group } & \multicolumn{2}{|c|}{ Healthy children group } & \multirow[t]{2}{*}{$P$-value } \\
\hline & Mean & Median & Mean & Median & \\
\hline \multicolumn{6}{|l|}{ Physical domain: } \\
\hline 1. Do you have dyspnea? & 2.1 & 2.0 & 1.3 & 1.0 & $<0.0001$ \\
\hline 2. Do you have palpitations? & 3.4 & 3.0 & 1.3 & 1.0 & $<0.0001$ \\
\hline 3. Do you have pain behind your breastbone? & 2.2 & 2.0 & 1.3 & 1.0 & $<0.0001$ \\
\hline 4. Do you ever faint? & 1.9 & 2.0 & 1.2 & 1.0 & $<0.0001$ \\
\hline $\begin{array}{l}\text { 5. Do you seem to pass urine more frequently than } \\
\text { usual? }\end{array}$ & 1.8 & 2.0 & 1.4 & 1.0 & $<0.0001$ \\
\hline 6. Do you ever have a blurred vision? (e.g. scotoma) & 2.3 & 2.0 & 1.8 & 2.0 & 0.0077 \\
\hline $\begin{array}{l}\text { 7. Do you think you are more pale than your friends } \\
\text { or do you tend to become pale suddenly? }\end{array}$ & 1.9 & 2.0 & 1.5 & 1.0 & 0.0010 \\
\hline $\begin{array}{l}\text { 8. Do you experience situations in which you sweat } \\
\text { more than your friends? }\end{array}$ & 2.3 & 2.0 & 1.5 & 1.0 & $<0.0001$ \\
\hline 9. Do you ever feel nauseous? & 2.0 & 2.0 & 1.7 & 1.0 & 0.0160 \\
\hline 10. Do you have headaches? & 2.7 & 3.0 & 2.6 & 3.0 & 0.6726 \\
\hline 11. Do you have stomach aches? & 2.3 & 2.0 & 2.4 & 3.0 & 0.3686 \\
\hline $\begin{array}{l}\text { 12. Do you sometimes feel suddenly cold without } \\
\text { a reason? }\end{array}$ & 2.1 & 2.0 & 1.5 & 1.0 & 0.0002 \\
\hline 13. Do you think you are weaker than your peers? & 3.0 & 3.0 & 1.6 & 1.0 & $<0.0001$ \\
\hline \multicolumn{6}{|l|}{ Psychological domain: } \\
\hline 1. Do you often cry? & 2.2 & 2.0 & 1.9 & 2.0 & 0.1659 \\
\hline 2. Is it easy to make you cry? & 2.7 & 2.0 & 2.2 & 2.0 & 0.0034 \\
\hline 3. Do you think you are more nervous than your peers? & 2.7 & 2.5 & 2.1 & 2.0 & $<0.0001$ \\
\hline 4. Do you think you are sadder than your peers? & 2.0 & 2.0 & 1.6 & 1.0 & 0.0075 \\
\hline 5. Do you think you are happier than your peers? & 2.8 & 3.0 & 2.7 & 3.0 & 0.7341 \\
\hline 6. Do you think you are more lonely than your peers? & 1.8 & 2.0 & 1.6 & 1.0 & 0.1810 \\
\hline 7. Can you count on your friends? & 4.3 & 4.0 & 4.3 & 5.0 & 0.7688 \\
\hline
\end{tabular}

Duration of arrhythmia (from the time of diagnosis until the time of questionnaire completion) and SVT type (AVRT, AVNRT, AET) had no impact on the parameters of the QoL.

In the SVT group the correlation was assessed between general satisfaction with the QoL and the scores obtained in the PARS questionnaire. Higher QoL with regard to Phd and Psd of PARS relate to generally higher evaluation of the QoL (WHOQOL); for Phd: $R s=-0.201, p=0.0068$; for Psd: $R s=-0.276, p=0.0002$. The correlation was also observed between Phd of the PARS questionnaire and Phd of the WHOQOL-BREF assessment: $R s=-0.5199, p<0.0001$. Similar results were obtained between Psd of the PARS questionnaire and Psd of the WHOQOL-BREF assessment: Rs = $-0.5782, p<0.0001$.

\section{Discussion}

The results of our study indicate that the QoL of SVT patients is worse than that of the healthy children. Both medical and psychological parameters of the QoL are lower in the group of SVT children. Healthy children reported significantly greater contentment with regard to general satisfaction with the QoL and general satisfaction with the health condition. No differences were observed in SRD referring to such areas as personal relationships or social support. Also, no differences were observed in terms of EnD, which concerns, among others, such areas as financial resources, freedom/safety, abilities to obtain new information and skills, transport and home environment and physical environment. The high level of satisfaction proves no relation between 
disease incidence and the above environmental areas.

This study demonstrates that the limitations resulting from the disease significantly lower the QoL of the children with SVT: the necessity to take medications as well as arrhythmia symptoms may have an additional negative impact on QoL, especially in terms of physical activities.

There are only a few available studies regarding evaluation of the QoL of pediatric patients with a diagnosed SVT, and these studies are based on a significantly lower number of patients in comparison with our study group.

Pulgaron et al. assessed the quality of life and psychosocial functioning of children with cardiac arrhythmias; however, the inclusion criterion in this study was the diagnosis of any type of cardiac arrhythmia [15]. Also, in the studied group 35\% of the patients previously underwent surgery related to their heart condition [15]. In our study we gathered a group of children with a diagnosed SVT, without organic heart condition or other chronic diseases that might impede the quality of life, with the aim of obtaining a more uniform group and reliable data. Apart from that, we gathered a group of healthy patients and we used them as a benchmark to analyze the scores obtained in the SVT group. Walfridsson et al. assessed the effects of SVT in adult patients on Health-Related QoL using reference groups [4], and this approach appears appropriate as it guarantees more reliable study results.

The available studies performed in pediatric patients show that the QoL of pediatric patients with cardiac arrhythmias is comparable to that of the children with chronic diseases [3]. Czosek et al. report that QoL scores in pediatric patients with SVT are not different from children with tetralogy of Fallot [7]. Moreover, Maryniak et al. reported that AVRT and AVNRT in children may have a negative impact on cognitive and emotional development [6].

Evaluation of the QoL has become a commonly applied procedure in clinical practice. Good treatment resulting in the improvement of patients' physical functioning or even their complete recovery does not always correspond with their psychological well-being or health perception. This is particularly often observed among cardiologic patients who are very concerned about their heart condition. Adult patients with cardiac conditions may suffer from depression and anxiety and may require psychotherapeutic support [16]. Disease effects on QoL are also perceived in other areas of medicine, and thus researchers develop either novel specific questionnaires adapted to particular groups of patients [17] or questionnaires evaluating particular aspects of psycho-social functioning, such as the Hospital Anxiety and Depression Scale (HADS) [18].

Currently, the QoL evaluation should be based on a generic questionnaire supplemented with a specific questionnaire for a studied group. For our study we used a short version of the WHOQOL-BREF questionnaire, which is an international, cross-culturally valid, universal and commonly applied instrument allowing for a comprehensive evaluation of the basic parameters of the QoL. It is an acknowledged tool, referred to in the subject literature [19]. It is used by investigators to assess the QoL not only in adults but also in children and teenagers [20, 21]. Currently, one of the existing and approved, specific questionnaires for children with cardiac conditions is the PedsQL Cardiac Module $[9,10]$, which was validated by Uzark et al. in pediatric patients with structural heart disease. This questionnaire is unfortunately not available in a Polish language version. The other one is the Pediatric Cardiac Quality of Life Inventory [11-13], adapted to cardiologic patients, also not available in Polish. Investigators try to create their own questionnaires adapted to children with cardiac arrhythmias. One such questionnaire is the Cardiac Arrhythmia Quality of Life for Youths, on the basis of which the authors evaluated the QoL and psychosocial functioning of pediatric patients with arrhythmia [15]. In our study we also attempted to develop our own specific questionnaire, adjusted to the studied group. Thus the Pediatric Arrhythmia Related Score questionnaire was created, which evaluates individual feelings and observations and which was used as a supplementary tool to perform our analysis.

On the basis of the performed QoL analysis, we suppose that the PARS questionnaire might be a useful tool that supplements the generic questionnaire. On the basis of the PARS questionnaire it was observed that patients with SVT much more frequently reported negative physical feelings and as a result they felt physically weaker than their healthy peers. Similarly, on the basis of the WHOQOL-BREF questionnaire we noted significantly higher quality of life within Phd among healthy children.

When analyzing psychological aspects, comparable results were also obtained regarding both questionnaires. Comparative analysis on the basis of the PARS questionnaire showed that the patients with SVT in comparison with healthy children were more nervous, sadder, less cheerful and, furthermore, they believed that more things made them cry when they compared themselves to healthy peers. On the basis of the WHOQOL-BREF questionnaire it was noted that more frequently they had negative emotions, such as unhappiness, fear, misery and depression, had worse self-es- 
teem and ability to concentrate than healthy children and generally worse QoL with regard to Psd.

General questionnaires provide more time-stable results and may not identify small but significant changes in the QoL. Questions in specific questionnaires address only those subjects which are more relevant in the population for which this questionnaire has been designed. They are more sensitive to changes and may reveal improvement of the QoL, even if the general questionnaire fails to prove that. We speculate that the PARS questionnaire may prove a more useful and sensitive tool than WHOQOL-BREF when evaluating the influence of ablation on patients' quality of life. In order to prove that, further analyses in the SVT group are necessary.

It should be stressed that 181 out of 183 patients included in our analysis underwent a successful RF ablation procedure and therefore further analysis and assessments are needed in order to evaluate whether ablation performed in our patients positively affects their psychophysical functioning.

Patients with SVT experience a lot of negative feelings, and it is important to assess how arrhythmia or the limitations related to the disease and its treatment impact the patient's physical, emotional and social well-being. These features are particularly useful in everyday clinical practice as the questionnaires may identify deterioration of the QoL, which can be difficult to observe during the course of routine check-ups. It appears useful to single out the patients for whom ablation treatment would be particularly recommended (medical and psychological reasons).

The authors acknowledge the following limitations of the performed study:

- although the presented group of patients is the most numerous pediatric SVT group described so far in the available literature concerning quality of life assessment, it is still quite a small group;

- the study was performed in one research center; - the study does not include the whole age range of the patients who undergo ablation treatments; due to the type of the selected method only children under 7 years of age were included in the study.

In conclusion, the QoL in children with SVT is significantly lower in comparison with healthy children. Both medical and psychological parameters of the QoL in the group of SVT children are lower.

The diagnosis of SVT has no influence on the social and environmental areas of the QoL. The PARS questionnaire appears to be a useful tool as a disease-specific quality of life instrument that supplements the generic questionnaire for QoL evaluation in children with SVT. It is a comparable instrument to WHOQOL-BREF for evaluation of physical and psychological functioning of SVT patients.

\section{Conflict of interest}

The authors declare no conflict of interest.

\section{References}

1. Hanash CR, Crosson JE. Emergency diagnosis and management of pediatric arrhythmias. J Emerg Trauma Shock 2010; 3: 251-60.

2. Doniger SJ, Sharieff GQ. Pediatric dysrhythmias. Pediatr Clin North Am 2006; 53: 85-105.

3. Strieper M, Leong T, Bajaj T, Huckaby J, Frias P, Campbell R. Does ablation of supraventricular tachycardia in children with a structurally normal heart improve quality of life? Congenit Heart Dis 2010; 5: 587-93.

4. Walfridsson $U$, Strömberg A, Janzon $M$, Walfridsson $H$. Wolff-Parkinson-White syndrome and atrioventricular nodal re-entry tachycardia in a Swedish population: consequences on health-related quality of life. Pacing Clin Electrophysiol 2009; 32: 1299-306.

5. Bubien RS, Knotts-Dolson SM, Plumb VJ, Kay GN. Effect of radiofrequency catheter ablation on health-related quality of life and activities of daily living in patients with recurrent arrhythmias. Circulation 1996; 94: 1585-91.

6. Maryniak A, Bielawska A, Bieganowska K, MiszczakKnecht M, Walczak F, Szumowski L. Does atrioventricular reentry tachycardia (AVRT) or atrioventricular nodal reentry tachycardia (AVRT) in children affect their cognitive and emotional development? Pediatr Cardiol 2013; 34: 893-7.

7. Czosek RJ, Cassedy AE, Wray J, et al. Quality of life in pediatric patients affected by electrophysiologic disease. Heart Rhythm 2015; 12: 899-908.

8. Delamater AM, Jent JF. Cardiovascular disease. In: Handbook of pediatric psychology. Roberts MC, Steele RG (eds). The Guilford Press, New York 2009; 381-91.

9. Uzark K, Jones K, Burwinkle TM, Varni JW. The pediatric quality of life inventory in children with heart disease. Prog Ped Card 2003; 18: 141-8.

10. Uzark K, Jones K, Slusher J, Limbers CA, Burwinkle TM, Varni JW. Quality of life in children with heart disease as perceived by children and parents. Pediatrics 2008; 121: 1060-7.

11. Marino BS, Shera D, Wernovsky G, et al. The development of the pediatric cardiac quality of life inventory: a quality of life measure for children and adolescents with heart disease. Qual Life Res 2008; 17: 613-26.

12. Wray J, Franklin R, Brown K, Cassedy A, Marino BS. Testing the pediatric cardiac quality of life inventory in the United kingdom. Acta Paediatr 2013; 102: 68-73.

13. Wray J, Franklin R, Brown K, Blyth J, Marino BS. Linguistic validation of a disease-specific quality of life measure for children and teenagers with cardiac disease. Cardiol Young 2012; 22: 13-7.

14. World Health Organization. Division of Mental Health. WHOQOL-BREF - introduction, administration, scoring and generic version of the assessment: field trial version, December 1996. Geneva, WHO 1996.

15. Pulgaron ER, Wile D, Schneider K, Young ML, Delamater AM. Quality of life and psychosocial functioning of children with cardiac arrhythmias. Cardiol Young 2013; 23: 82-8.

16. Moryś JM, Bellwon J, Höfer S, Rynkiewicz A, Gruchała M. Quality of life in patients with coronary heart disease after myocardial infarction and with ischemic heart failure. Arch Med Sci 2016; 12: 326-33. 
17. Dudzińska M, Tarach JS, Burroughs TE, et al. Validation of the Polish version of Diabetes Quality of Life - Brief Clinical Inventory (DQL-BCl) among patients with type 2 diabetes. Arch Med Sci 2014; 10: 891-98.

18. Watrowski R, Rohde A. Validation of the Polish version of the Hospital Anxiety and Depression Scale in three populations of gynecologic patients. Arch Med Sci 2014; 10: $517-24$

19. Skevington SM, Lotfy M, O'Connell KA; WHOQOL Group. The World Health Organization's WHOQOL-BREF quality of life assessment: psychometric properties and results of the international field trial. A report from the WHOQOL group. Qual Life Res 2004; 13: 299-310.

20. Rana P, Mishra D. Quality of life of unaffected siblings of children with chronic neurological disorders. Indian J Pediatr 2015; 82: 545-8.

21. Kaheni S, Yaghobian M, Sharefzadah GH, Vahidi A, Ghorbani $H$, Abderahemi A. Quality of life in children with beta-thalassemia major at center for special diseases. Iran J Ped Hematol Oncol 2013; 3: 108-13. 


\section{Appendix 1}

The questions included in the WHOQOL-BREF assessment:

1. How would you rate your quality of life?

2. How satisfied are you with your health?

3. To what extent do you feel that physical pain prevents you from doing what you need to do?

4. How much do you need any medical treatment to function in your daily life?

5. How much do you enjoy life?

6. To what extent do you feel your life to be meaningful?

7. How well are you able to concentrate?

8. How safe do you feel in your daily life?

9. How healthy is your physical environment?

10. Do you have enough energy for everyday life?

11. Are you able to accept your bodily appearance?

12. Have you enough money to meet your needs?

13. How available to you is the information that you need in your day-to-day life?

14. To what extent do you have the opportunity for leisure activities?

15. How well are you able to get around?

16. How satisfied are you with your sleep?

17. How satisfied are you with your ability to perform your daily living activities?

18. How satisfied are you with your capacity for work?

19 . How satisfied are you with yourself?

20. How satisfied are you with your personal relationships?

21. How satisfied are you with your sex life?

22. How satisfied are you with the support you get from your friends?

23. How satisfied are you with the conditions of your living place?

24. How satisfied are you with your access to health services?

25. How satisfied are you with your mode of transportation?

26. How often do you have negative feelings, such as blue mood, despair, anxiety, depression? 


\section{Appendix 2}

The questions included in the questionnaire of individual patient's feeling and observations: (Pediatric Arrhythmia Related Score - PARS)

Physical domain:

1. Do you have dyspnea?

2. Do you have palpitations?

3. Do you have pain behind your breastbone?

4. Do you ever faint?

5. Do you seem to pass urine more frequently than usual?

6. Do you ever have a blurred vision (e.g. scotoma)?

7. Do you think you are more pale than your friends or do you tend to become pale suddenly?

8. Do you experience situations in which you sweat more than your friends?

9. Do you ever feel nauseous?

10. Do you have headaches?

11. Do you have stomach aches?

12. Do you sometimes feel suddenly cold without a reason?

13. Do you think you are weaker than your peers?

Medical satisfaction domain:

1. Would you like to learn more details about your disease?

2. Would you like to learn more details about the treatment?

3. Do you think that ablation treatment is effective?

4. Are you afraid of medical appointments?

5. Are you afraid of such examinations as ECG or echocardiography?

6. Are you afraid of ablation treatment?

7. Are you afraid of a blood draw?

8. Do you understand the idea of ablation treatment?

9. Have you ever hidden your disease symptoms from your doctor?

10. Have you ever not followed doctor's recommendations?

11. Do you always follow all doctor's recommendations regarding the intake of medicines?

12. Do you limit your physical exercise if this is recommended by your doctor?

Psychological domain:

1. Do you often cry?

2. Is it easy to make you cry?

3. Do you think you are more nervous than your peers?

4. Do you think you are sadder than your peers?

5. Do you think you are happier than your peers?

6. Do you think you are more lonely than your peers?

7. Can you count on your friends? 